\title{
Profiling of prognostic alternative splicing in melanoma
}

\author{
FU-CHAO MA ${ }^{1}$, RONG-QUAN HE ${ }^{1}$, PENG LIN ${ }^{2}$, JIN-CAI ZHONG ${ }^{1}$, \\ JIE MA ${ }^{1}$, HONG YANG $^{2}$, XIAO-HUA HU ${ }^{1}$ and GANG CHEN ${ }^{3}$ \\ ${ }^{1}$ Department of Medical Oncology, ${ }^{2}$ Ultrasonics Division of Radiology Department, \\ ${ }^{3}$ Department of Pathology, First Affiliated Hospital of Guangxi Medical University, \\ Nanning, Guangxi 530021, P.R. China
}

Received April 19, 2018; Accepted April 12, 2019

DOI: $10.3892 / \mathrm{ol} .2019 .10453$

\begin{abstract}
Alternative splicing can lead to the coding of proteins that act as promoters of cancer, which is associated with the progression of cancer. However, to the best of our knowledge, no systematic survival analysis of alternative splicing in melanoma has previously been reported. The present study conducted an in-depth analysis of integrated alternative splicing events detected in 96 patients with melanoma using data obtained from The Cancer Genome Atlas. Prognostic models and an alternative splicing correlation network were built for patients with melanoma. A total of 41,446 mRNA splicing events were detected in 9,780 genes and 2,348 alternative splicing events were identified to be significantly associated with overall survival of patients with melanoma. Of all the events used in the prognostic model, the model with alternate terminator alternative splicing events exhibited the highest efficiency for evaluating the outcome of patients with melanoma, with an area under the curve of 0.902 . The present study identified prognostic predictors for melanoma and revealed alternative splicing networks in melanoma that could indicate underlying mechanisms.
\end{abstract}

\section{Introduction}

Melanoma, the most aggressive type of skin cancer, develops from melanocytes and accounts for $4 \%$ of all cancer types diagnosed in adolescents worldwide (1-3). While the incidence rate of melanoma is lower than other types of skin cancer, it can grow rapidly and metastasize when it is not detected and treated early (4-6). Therefore, late-stage melanoma can be difficult to treat and fatal (7). The 5-year survival rate ranges between 15 and $60 \%$ depending on the stage of the melanoma $(4,8)$.

Correspondence to: Professor Xiao-Hua Hu, Department of Medical Oncology, First Affiliated Hospital of Guangxi Medical University, 6 Shuangyong Road, Nanning, Guangxi 530021, P.R. China

E-mail: gxmuhxh@163.com

Key words: prognostic, alternative splicing, melanoma, The Cancer Genome Atlas, overall survival
It is understood that disordered gene expression serves an important role in the development of cancer (9). Gene expression profiling can provide evidence to determine the diagnosis and identify prognostic markers or novel therapeutic targets in cancer $(10,11)$. However, a limited number of previous studies have investigated the role of gene expression by examining alternative splicing events $(9,12,13)$.

Alternative splicing can lead to the coding of several types of proteins. During alternative splicing, specific exons of a gene can be included or excluded and the alternatively spliced mRNAs are then translated into proteins that exhibit different biological functions (12-14). Alternative splicing regulates gene expression and serves a key role in this process (9). Defects in mRNA splicing can result in aberrant splicing, in addition to oncogenic processes (15-17). A number of previous studies have demonstrated that splicing defects act as promoters of cancer (16,18-20). Furthermore, different splicing factors may cause specific cancer-promoting isoforms.

It has been demonstrated that alternative splicing exhibits prognostic value in patients with lung (21), ovarian (22) and breast cancer (23), and glioblastoma (24). However, to the best of our knowledge, a systematic survival analysis of alternative splicing in melanoma has not been previously performed, and thus is urgently required. The aim of the present study was to determine prognostic alternative splicing events in melanoma using RNA sequencing (RNA-seq) data from The Cancer Genome Atlas (TCGA).

\section{Materials and methods}

Alternative splicing data collection. Melanoma cohort RNA-seq data (25) were downloaded from TCGA data portal (tcga-data.nci.nih.gov/tcga). Data of 96 melanoma cases with clinicopathological information were obtained to explore the changes of alternative splicing events in association with the carcinogenesis and prognosis of melanoma. To analyze the alternative splicing profiles for each patient, a SpliceSeq tool (version 2.1) (26), which is a java application, was used in the melanoma cohort to evaluate the splicing patterns of mRNA. The percent spliced in (PSI) value was calculated to quantify alternative splicing events ranging between 0 and 1 in seven types of alternative splicing events, including exon skip (ES), alternate terminator (AT), mutually exclusive (ME) 
exons, retained intron (RI), alternate promoter (AP), alternate acceptor (AA) site and alternate donor (AD) site.

Survival analysis. The melanoma clinical data were downloaded from TCGA database. Only patients with an overall survival (OS) time $>90$ days were enrolled in the study. A total of 96 patients with melanoma were included in the present study. The patients were divided into two groups (high- and low-risk groups) according to the median cut-off value (0.078). Kaplan-Meier and Univariate Cox regression analyses were used to analyze associations between splicing factor genes and survival, and multivariate Cox regression was performed to remove non-independent predictors. To compare the efficiencies of each prediction model, receiver operator characteristic (ROC) curves were generated using the survival receiver ROC package (version 1.0.3; cran.r-project.org/web/packages/survivalROC/index.html) in R software (version 3.3.0; cran.r-project.org/bin/windows/base/old/3.3.0). To ascertain the predictive ability, the area under the curve (AUC) was calculated for each model.

Statistical analysis. The UpSet package (version 1.3.3; CRAN.R-project.org/package $=$ UpSetR) in R software was used to quantitatively analyze the alternative splicing intersections for all seven types of alternative splicing events (27). The most significant alternative splicing events were selected to generate a gene network using the Reactome FI Cytoscape 3.6.0 plugin to search for the key hub genes of survival associated with the alternative splicing genes (28). Cytoscape 3.6.0 was then used to generate correlation plots. Spearman's rank correlation coefficient was used to analyze the correlation between the PSI values and the splicing factor gene expression to identify potential prognostic biomarkers for melanoma.

\section{Results}

Overview of alternative splicing events in TCGA melanoma cohort. An in-depth analysis of integrated alternative splicing events was conducted for 96 patients with melanoma with an OS time $>90$ days. In the melanoma cohort, 41,446 mRNA splicing events were detected in 9,780 genes, which consisted of 15,892 ESs in 6,160 genes, 8,267 ATs in 3,614 genes, 8,135 APs in 3,273 genes and 3,315 AAs in 2,350 genes, 2,968 ADs in 2,070 genes, 2,656 RIs in 1,780 genes and 177 MEs in 175 genes. The results suggested that several mRNA splicing events occur in one gene and $\sim 38 \%$ of the alternative splicing events were ES events, followed by $19.9 \%$ AT events and 19.6\% AP events (Fig. 1).

Survival-associated alternative splicing events in TCGA melanoma cohort. Every alternative splicing event in TCGA melanoma cohort was evaluated for its association with survival. The patients with melanoma were divided into two groups (high- and low-risk groups) based on the median PSI value. A total of 2,348 survival-associated alternative splicing events (data not shown) were detected in melanoma $(\mathrm{P}<0.05)$. The top ten most significant events in each type of alternative splicing event were selected. More than one alternative splicing event could occur in one gene, therefore, an UpSet plot was generated to visualize the intersecting sets. As demonstrated

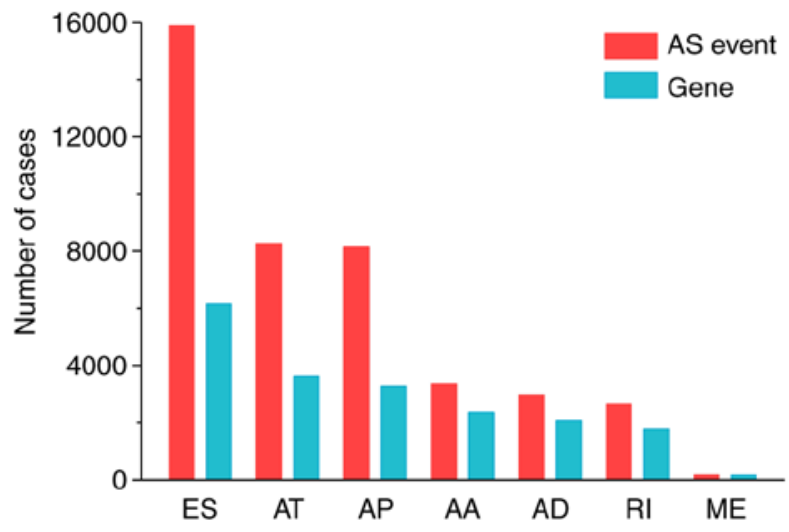

Figure 1. Number of AS events and the associated genes in 96 patients with melanoma. AS, alternative splicing; ES, exon skip; AT, alternate terminator; AP, alternate promoter; AA, alternate acceptor; AD, alternate donor; $\mathrm{RI}$, retained intron; ME, mutually exclusive.

in Fig. 2, up to three types of splicing event were identified in one gene; however, the majority of the survival-associated alternative splicing events were from one gene and these were all associated with patient survival. Furthermore, a gene network was created by Cytoscape analysis. This revealed important cancer pathways in melanoma by the identification of the following hub genes: RNA polymerase II subunit I, epidermal growth factor receptor, nucleoporin 160, ribosomal protein S15A and ribosomal protein S3A (Fig. 3). Notably, numerous favorable prognostic factors were identified in the survival-associated alternative splicing events.

Prognostic predictors for patients with melanoma. The OS time of patients with melanoma ranged between 126 and 1,785 days. The top ten most significant events in the seven types of alternative splicing events were selected to determine the independent prognostic factors for patients with melanoma. Multivariate Cox regression analysis with a prognostic model construction was used to analyze the seven types of alternative splicing events separately, as demonstrated in Fig. 4. Kaplan-Meier curves of the prognostic predictors were generated for AA, AD, AP, AT, ES, ME and RI. The red line indicates the high-risk group and the blue line indicates the low-risk group. The seven predictors were integrated for all types of alternative splicing events in order to analyze the final prognostic model for patients with melanoma (Fig. 5). ROC curves were generated for the individual types of splicing event (Fig. 6) and for a combination of all types of splicing events (Fig. 7), and AUC analysis was used to evaluate the efficiency of the prognostic models. In the high-risk and low-risk groups, the median OS times were 472 and 1,354 days, respectively $(\mathrm{P}<0.0001 ;$ Fig. 5). Of all the models, the model with the AT alternative splicing events exhibited the highest efficiency for evaluating outcomes for patients with melanoma (Fig. 6D). The AUC value of the AT alternative splicing events was 0.902 , followed by the AP model with an AUC value of 0.887 (Fig. 6C) and the RI model with an AUC value of 0.809 (Fig. 6G).

Alternative splicing correlation network in melanoma. A total of eight splicing factors, heterogeneous nuclear 


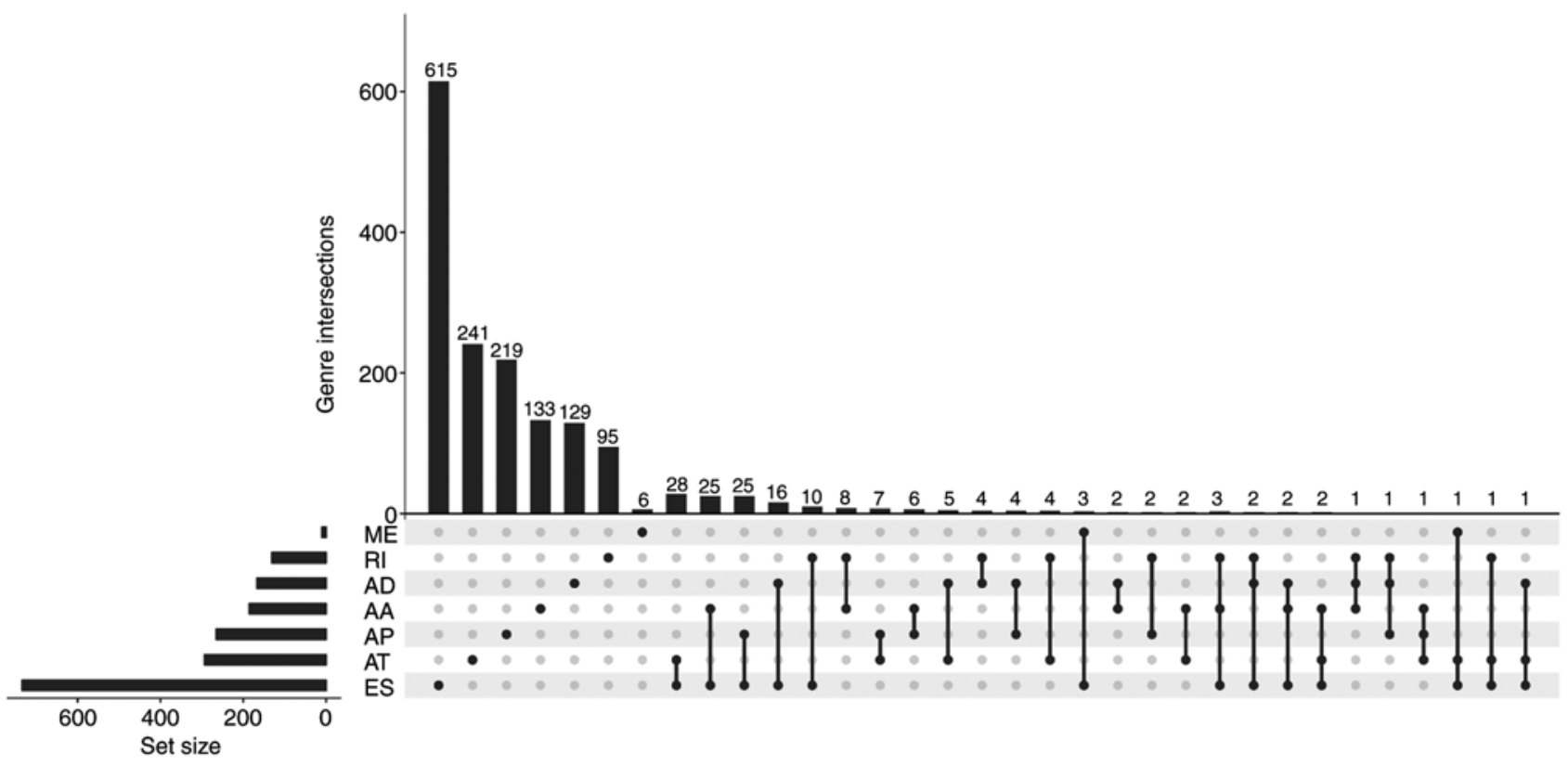

Figure 2. UpSet plot of alternative splicing events in melanoma. UpSet plot of the interactions of the alternative splicing events associated with survival in melanoma. Up to three types of alternative splicing events associated with patient survival were identified to occur in one gene. ES, exon skip; AT, alternate terminator; AP, alternate promoter; AA, alternate acceptor; $\mathrm{AD}$, alternate donor; RI, retained intron; ME, mutually exclusive.

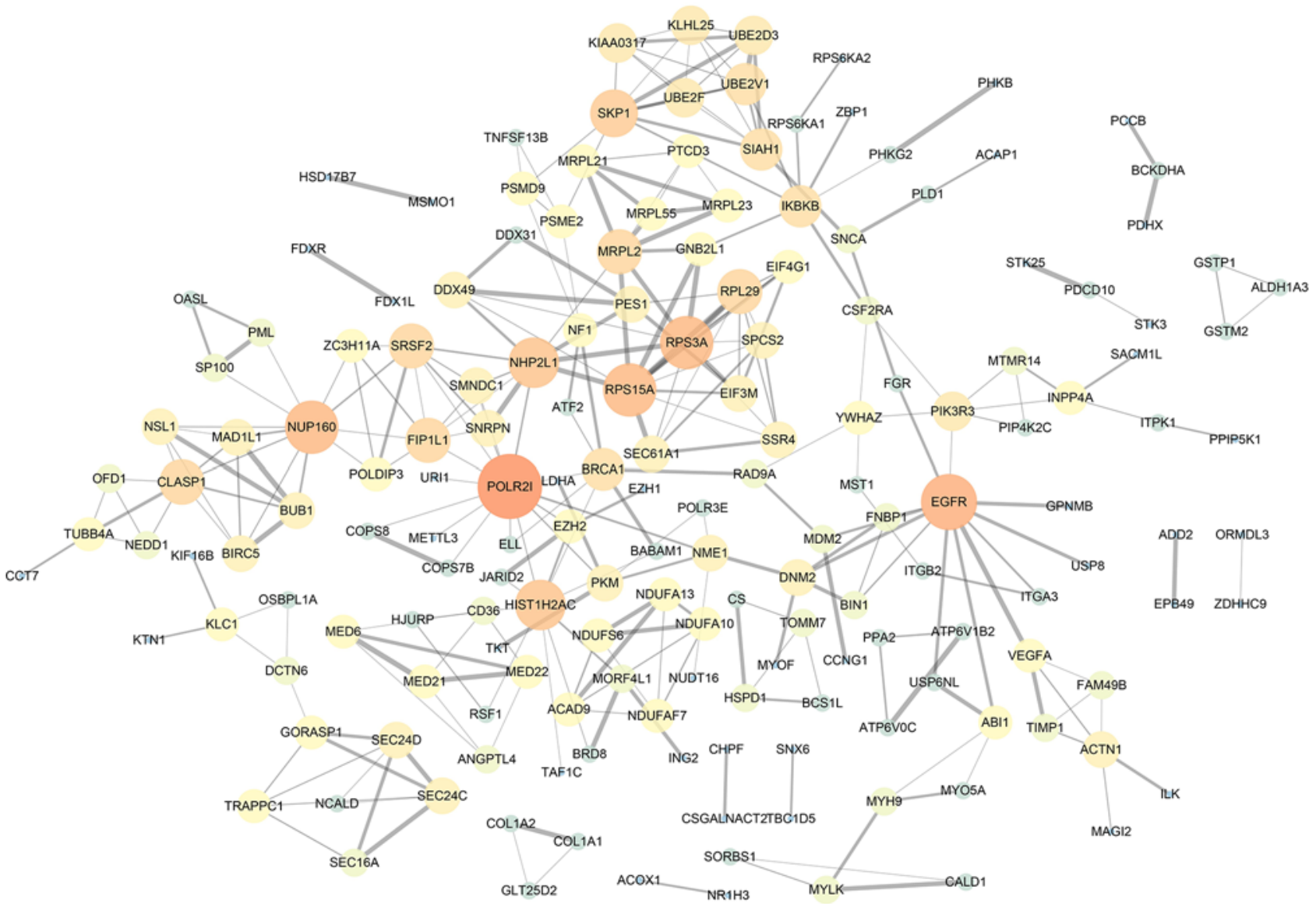

Figure 3. Gene network of alternative splicing events in melanoma. Gene network of survival-associated alternative splicing in melanoma generated using Cytoscape. The size and brightness of the circles represent the degree of connection. The larger and brighter circles are the hub genes in the network.

ribonucleoprotein $\mathrm{L}$, serine and arginine rich splicing factor 9, Y-box binding protein 1, poly(rC) binding protein 2, heterogeneous nuclear ribonucleoprotein $\mathrm{C}(\mathrm{C} 1 / \mathrm{C} 2)$, heterogeneous nuclear ribonucleoprotein $\mathrm{A} 1$, serine and arginine rich 

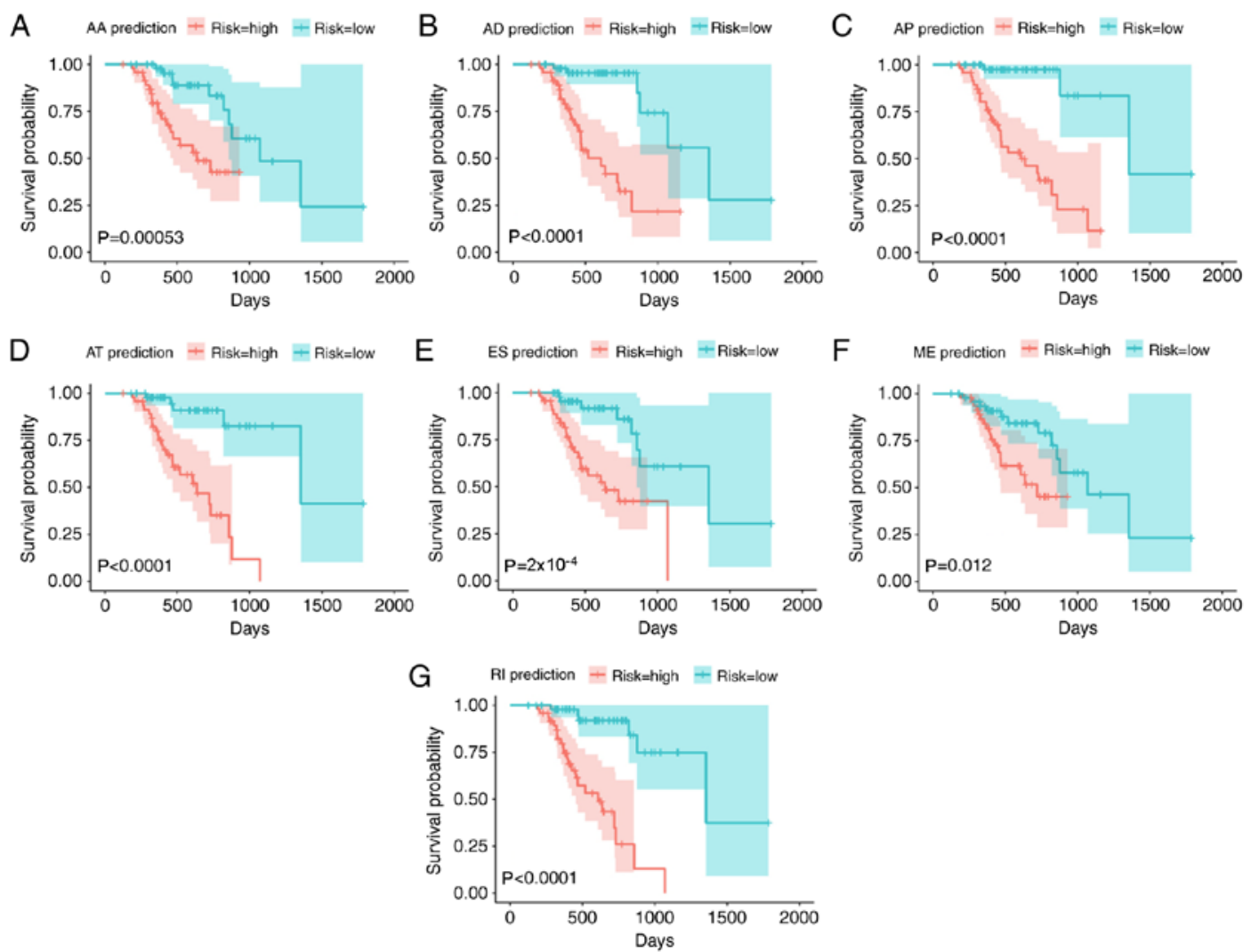

Figure 4. Kaplan-Meier curves of the prognostic predictors for patients with melanoma. Kaplan-Meier curves of the prognostic predictors were generated for each type of alternative splicing event in melanoma. Kaplan-Meier curves of the prognostic predictors were generated for (A) AA, (B) AD, (C) AP, (D) AT, (E) ES, (F) ME and (G) RI. The red line indicates the high-risk group and the blue line indicates the low-risk group. AA, alternate acceptor; AD, alternate donor; AP, alternate promoter; AT, alternate terminator; ES, exon skip; ME, mutually exclusive; RI, retained intron.
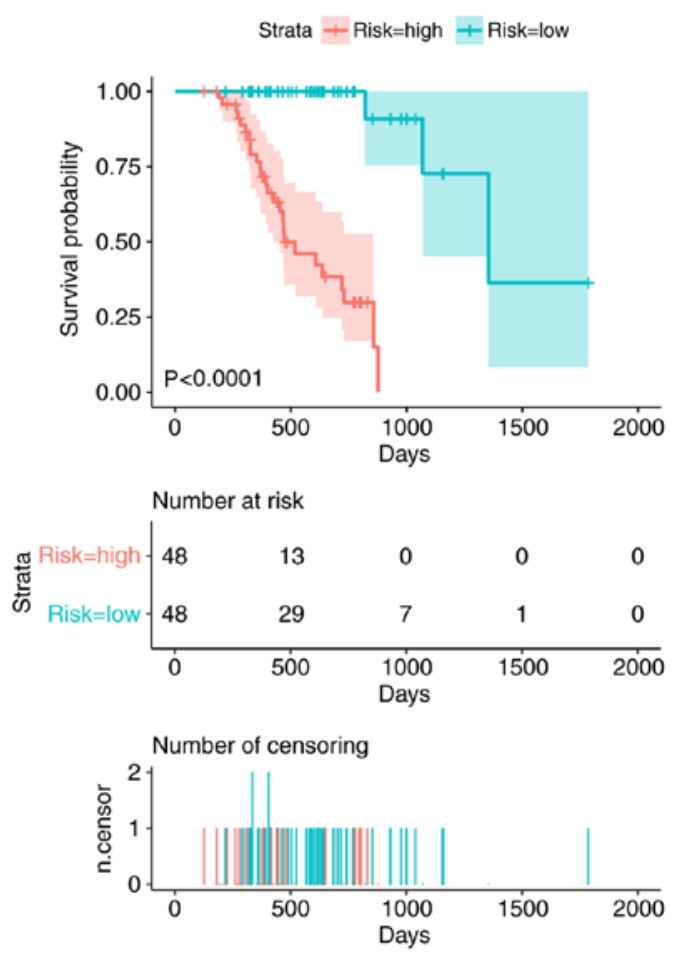

Figure 5. Kaplan-Meier curve of integrated prognostic predictors for all types of alternative splicing events for melanoma. Patients were divided into a high-risk group $(n=48)$ and a low-risk group $(n=48)$ based on prognostic predictors. Patients with survival $<1000$ days from surgery were censored. And the number of censoring mainly concentrated within 1,000 days. The red line indicates the high-risk group and the blue line indicates the low-risk group. splicing factor 3 and serine and arginine rich splicing factor 4, were identified. As demonstrated in Fig. 8, these splicing factors, represented by green dots, were markedly associated with the OS time of the patients in the survival analysis. The expression levels and PSI values of these eight splicing factors identified from the most significant alternative splicing events were used to conduct the correlation analysis in the melanoma cohort based on the significant correlation results $(\mathrm{P}<0.05)$. As presented in Fig. 8, 50 survival-associated alternative splicing events were identified in the splicing correlation network, including 31 adverse alternative splicing events (red dots) and 19 favorable alternative splicing events (blue dots). The most favorable prognostic alternative splicing events (blue dots) were positively correlated (blue line) with the expression of the eight splicing factors (green dots), while the majority of the adverse prognosis alternative splicing events (red dots) were negatively correlated (red line ) with the expression of the eight splicing factors.

\section{Discussion}

Previous studies have reported several aberrant alternative splicing events in melanoma (28-31). Evidence has demonstrated that alternative splicing serves an important role in the occurrence and development of melanoma. For example, jumonji domain containing 6 , arginine demethylase and lysine hydroxylase (JMJD6) regulates alternative splicing of p21 (RAC1) activated kinase 1, which is a key component of 

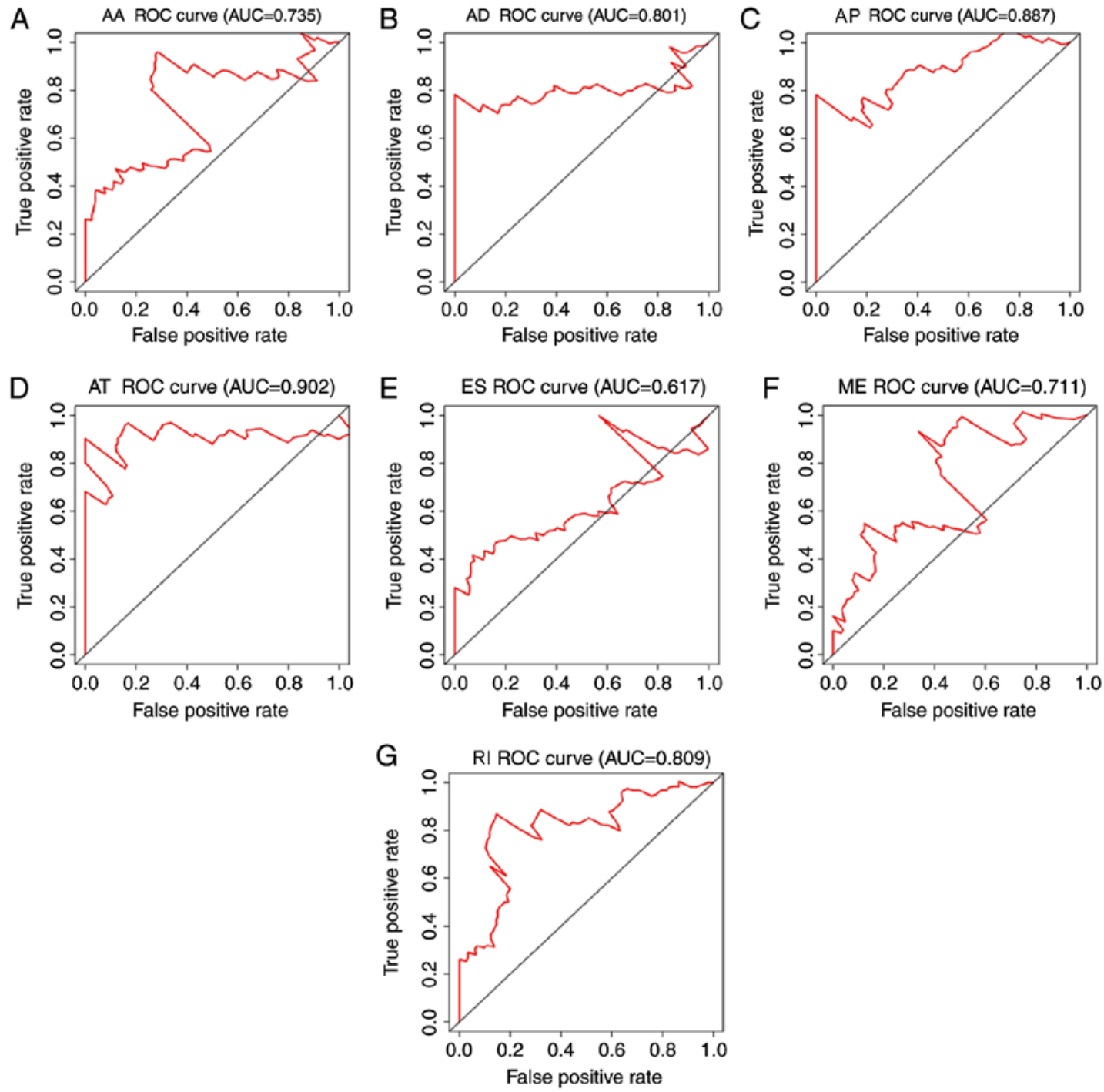

Figure 6. ROC curves with AUC of the prognostic predictors for each type of alternative splicing event in melanoma. ROC curves with AUC of the prognostic predictors for (A) AA, (B) AD, (C) AP, (D) AT, (E) ES, (F) ME and (G) RI are presented. ROC, receiver operating characteristic; AUC, area under the curve; ES, exon skip; AT, alternate terminator; AP, alternate promoter; AA, alternate acceptor; AD, alternate donor; RI, retained intron; ME, mutually exclusive.

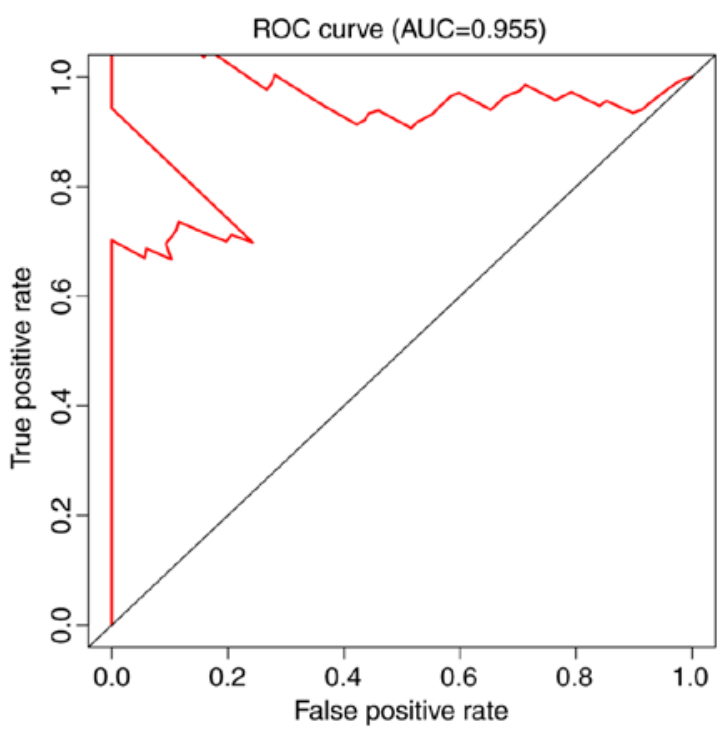

Figure 7. ROC curve with AUC of the prognostic predictors for all types of alternative splicing events in melanoma. ROC, receiver operating characteristic; AUC, area under the curve. mitogen-activated protein kinase signaling; therefore, JMJD6 may promote carcinogenesis of melanoma (29). Alternative splicing of CD44 mediated by U2 small nuclear RNA auxiliary factor 2 promotes melanoma metastasis (30). However, thorough studies of alternative splicing may provide further information regarding the oncogenes and tumor suppressor genes of melanoma. Splicing factor $3 b$ subunit 1 (SF3B1) is a key spliceosome component that was mutated in melanoma (30), chronic lymphocytic leukemia (32) and pancreatic cancer (33). Mutation of SF3B1 has been associated with good melanoma prognosis (31). A splicesome of human telomerase reverse transcriptase may control the activity of telomerase in melanoma. In the present study, 2,348 alternative splicing events were markedly associated with the OS time of patients with melanoma. The results of the present study are consistent with the findings reported in previous studies $(29,30)$. These previous studies provide information regarding alternative splicing and splicing factors in melanoma, which may result in the identification of potential biomarkers and the development of therapeutic targets. 


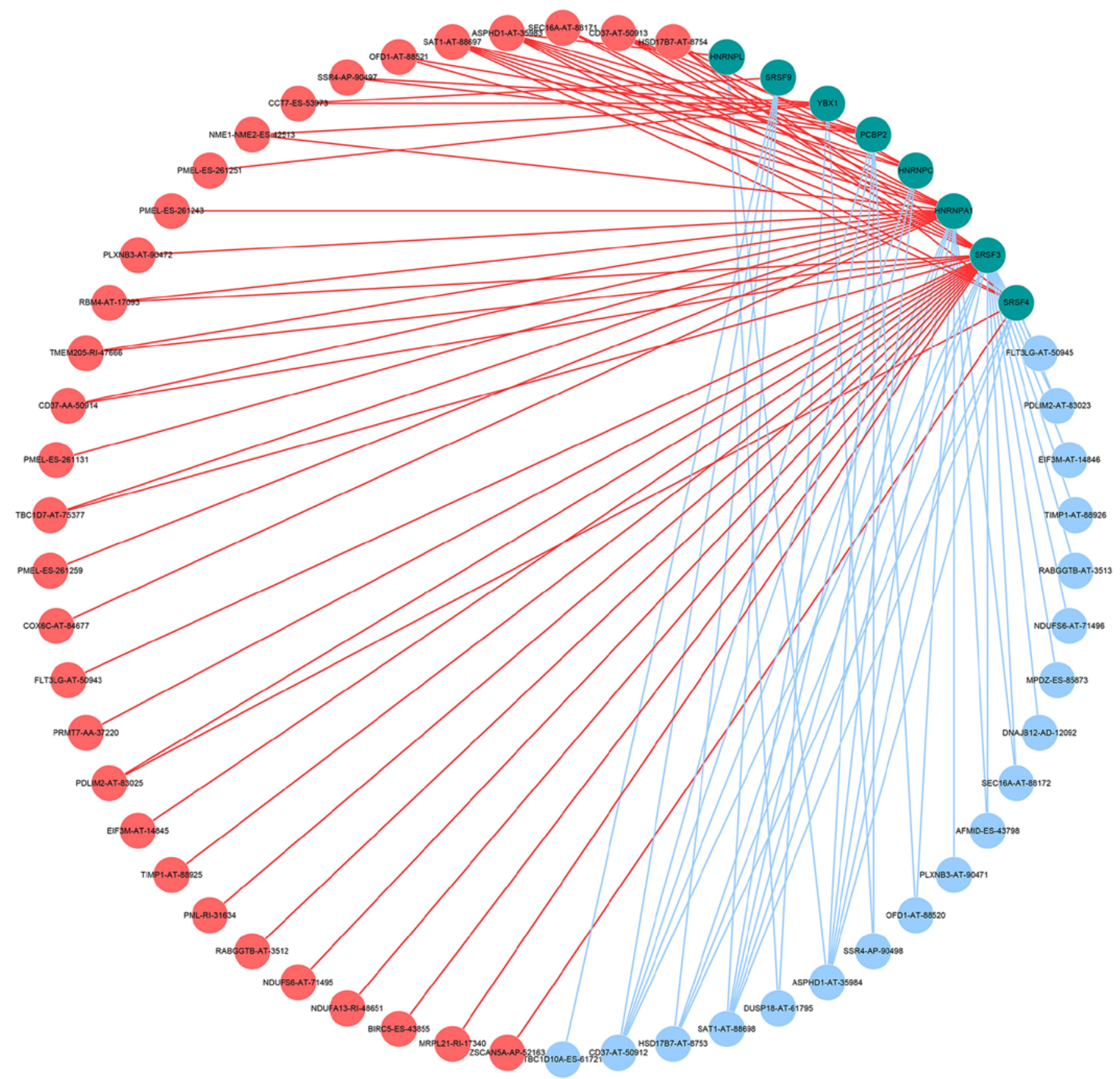

Figure 8. Splicing correlation network for melanoma. Blue dots indicate that the alternative splicing PSI was positively correlated with survival times. Red dots indicate that the alternative splicing PSI was negatively correlated with survival times. Green dots indicate the survival-associated splicing factors. The blue lines indicate a positive correlation between the expressions of the splicing factors with the PSI values of the alternative splicing events, while the red lines indicate a negative correlation. PSI, percent spliced in.

The development of high throughput sequencing technology has provided information regarding the features of alternative splicing $(34,35)$. It is challenging to identify the splicesomes associated with cancer that determine the splicing patterns in the disease. Aberrant patterns of splicing leads to unlimited proliferation of cancer (36). The motility and invasion of cancer cells can be enhanced by serine and arginine-rich splicing factor 1 , which modifies the expression of Ron-receptor tyrosine kinase (37). It was identified that vascular endothelial growth factor (VEGF) leads to angiogenesis in cancer. VEGF-A165, a splice variant of VEGF, is highly angiogenic; however, another splice variant of VEGF, VEGF-A165b, is downregulated in several types of cancer, such as colorectal cancer (38) and renal cell carcinoma (39). VEGF-A165b is associated with a poor prognosis of colorectal cancer (38). Although the role of splice variants is not completely understood, evidence indicates that they are strongly associated with specific types of cancer and exhibit potential prognostic value.

In the present study, alternative splicing features in 96 patients with melanoma were profiled using integrated survival analysis. A total of 41,446 alternative splicing events from 9,780 genes were detected and $38 \%$ were ES events, followed by AT and AP events. ROC curves and Cox regression 
models were used to evaluate alternative splicing patterns as early biomarkers for melanoma occurrence or prognosis. Among the seven types of alternative splicing events, the AT events exhibited the highest efficiency for differentiating good or poor outcomes in patients with melanoma. The AUC in this model was 0.902 , which is the highest value for all seven types of alternative splicing events, suggesting that it has the greatest potential for use as a prognostic marker for melanoma.

In addition, the present analysis highlighted the potential participation of splicing factors. The majority of the favorable prognosis alternative splicing events were positively correlated with the expression of splicing factors and the adverse prognosis alternative splicing events were negatively correlated with the expression of splicing factors. Expression of specific splicing factors may help determine if favorable or adverse alternative splicing events for prognosis require further validation by functional experiments. The present study investigated potential key splicing factors in melanoma and provided a background for understanding the role of splicesomes in melanoma.

In conclusion, the present study reported that alternative splicing events, which are associated with survival, may be used to generate a prognostic model for risk stratification in melanoma that could be applied in clinical practice. Furthermore, the present study provided novel insight into how pivotal splicing factors regulate aberrant alternative splicing. From the alternative splicing events and splicing factors that are associated with survival, several potential biomarkers or therapeutic targets were identified that require validation in future studies. Finally, a mechanism for deciphering alternative splicing events in tumorigenesis in melanoma was proposed.

\section{Acknowledgements}

Not applicable.

\section{Funding}

No funding was received.

\section{Availability of data and materials}

The datasets analyzed during the study are available from the corresponding author upon reasonable request.

\section{Authors' contributions}

GC and XHH designed the study. FCM, RQH, PL, JCZ, JM and $\mathrm{HY}$ conducted the experiments and analyzed the data. FM wrote the manuscript. All authors read and approved the manuscript and agree to be accountable for all aspects of the research in ensuring that the accuracy or integrity of any part of the work are appropriately investigated and resolved.

\section{Ethics approval and consent to participate}

Not applicable.

\section{Patient consent for publication}

Not applicable.

\section{Competing interests}

The authors declare that they have no competing interests.

\section{References}

1. Siegel RL, Miller KD and Jemal A: Cancer statistics, 2018. CA Cancer J Clin 68: 7-30, 2018.

2. Cui L, Li Y, Lv X, Li J, Wang X, Lei Z and Li X: Expression of MicroRNA-301a and its functional roles in malignant melanoma. Cell Physiol Biochem 40: 230-244, 2016.

3. Mahgoub T, Eustace AJ, Collins DM, Walsh N, O'Donovan N and Crown J: Kinase inhibitor screening identifies CDK4 as a potential therapeutic target for melanoma. Int $\mathrm{J}$ Oncol 47: 900-908, 2015

4. Finn L, Markovic SN and Joseph RW: Therapy for metastatic melanoma: The past, present, and future. BMC Med 10: 23, 2012.

5. Carpinteiro A, Beckmann N, Seitz A, Hessler G, Wilker B, Soddemann M, Helfrich I, Edelmann B, Gulbins E and Becker KA: Role of acid sphingomyelinase-induced signaling in melanoma cells for hematogenous tumor metastasis. Cell Physiol Biochem 38: 1-14, 2016.

6. Pagani E, Ruffini F, Antonini Cappellini GC, Scoppola A, Fortes C, Marchetti P, Graziani G, D'Atri S and Lacal PM: Placenta growth factor and neuropilin-1 collaborate in promoting melanoma aggressiveness. Int J Oncol 48: 1581-1589, 2016.

7. Houghton AN and Polsky D: Focus on melanoma. Cancer Cell 2: 275-278, 2002

8. Caporali S, Alvino E, Lacal PM, Levati L, Giurato G, Memoli D, Caprini E, Antonini Cappellini GC and D'Atri S: Targeting the $\mathrm{PI} 3 \mathrm{~K} / \mathrm{AKT} / \mathrm{mTOR}$ pathway overcomes the stimulating effect of dabrafenib on the invasive behavior of melanoma cells with acquired resistance to the BRAF inhibitor. Int J Oncol 49: 1164-1174, 2016.

9. David CJ, Chen M, Assanah M, Canoll P and Manley JL: HnRNP proteins controlled by c-Myc deregulate pyruvate kinase mRNA splicing in cancer. Nature 463: 364-368, 2010.

10. Zhang J, Shao J, Zhu L, Zhao R, Xing J, Wang J, Guo X, Tu S, $\mathrm{Han} \mathrm{B}$ and $\mathrm{Yu} \mathrm{K}$ : Molecular profiling identifies prognostic markers of stage IA lung adenocarcinoma. Oncotarget 8: 74846-74855, 2017.

11. Alaterre E, Raimbault S, Goldschmidt H, Bouhya S, Requirand G, Robert N, Boireau S, Seckinger, Hose D, Klein B and Moreaux J: CD24, CD27, CD36 and CD302 gene expression for outcome prediction in patients with multiple myeloma. Oncotarget 8: 98931-98944, 2017.

12. Kelemen O, Convertini P, Zhang Z, Wen Y, Shen M, Falaleeva M and Stamm S: Function of alternative splicing. Gene 344: 1-20, 2005.

13. Iqbal SM, Andavan GS and Lemmens-Gruber R: Differential modulation of fast inactivation in cardiac sodium channel splice variants by Fyn tyrosine kinase. Cell Physiol Biochem 37: 825-837, 2015.

14. Avin BA, Umbricht CB and Zeiger MA: Human telomerase reverse transcriptase regulation by DNA methylation, transcription factor binding and alternative splicing (Review). Int $\mathrm{J}$ Oncol 49: 2199-2205, 2016.

15. Bonomi S, Gallo S, Catillo M, Pignataro D, Biamonti G and Ghigna C: Oncogenic alternative splicing switches: Role in cancer progression and prospects for therapy. Int J Cell Biol 2013: 962038, 2013

16. Fackenthal JD and Godley LA: Aberrant RNA splicing and its functional consequences in cancer cells. Dis Model Mech 1: 37-42, 2008.

17. Xu Q, Gao J and $\mathrm{Li} Z$ : Identification of a novel alternative splicing transcript variant of the suppressor of fused: Relationship with lymph node metastasis in pancreatic ductal adenocarcinoma. Int J Oncol 49: 2611-2619, 2016.

18. Izaguirre DI, Zhu W, Hai T, Cheung HC, Krahe R and Cote GJ: PTBP1-dependent regulation of USP5 alternative RNA splicing plays a role in glioblastoma tumorigenesis. Mol Carcinog 51: 895-906, 2012. 
19. Dhir A and Buratti E: Alternative splicing: Role of pseudoexons in human disease and potential therapeutic strategies. FEBS J 277: 841-855, 2010.

20. Karni R, de Stanchina E, Lowe SW, Sinha R, Mu D and Krainer AR: The gene encoding the splicing factor SF2/ASF is a proto-oncogene. Nat Struct Mol Biol 14: 185-193, 2007.

21. Li Y, Sun N, Lu Z, Sun S, Huang J, Chen Z and He J: Prognostic alternative mRNA splicing signature in non-small cell lung cancer. Cancer Lett 393: 40-51, 2017.

22. Zhu J, Chen Z and Yong L: Systematic profiling of alternative splicing signature reveals prognostic predictor for ovarian cancer. Gynecol Oncol 148: 368-374, 2018.

23. Suo C, Hrydziuszko O, Lee D, Pramana S, Saputra D, Joshi H, Calza $S$ and Pawitan Y: Integration of somatic mutation, expression and functional data reveals potential driver genes predictive of breast cancer survival. Bioinformatics 31: 2607-2613, 2015.

24. Pal S, Bi Y, Macyszyn L, Showe LC, O'Rourke DM and Davuluri RV: Isoform-level gene signature improves prognostic stratification and accurately classifies glioblastoma subtypes. Nucleic Acids Res 42: e64, 2014.

25. Robertson AG, Shih J, Yau C, Gibb EA, Oba J, Mungall KL, Hess JM, Uzunangelov V, Walter V, Danilova L, et al: Integrative analysis identifies four molecular and clinical subsets in uveal melanoma. Cancer Cell 32: 204-220.e15, 2017.

26. Ryan MC, Cleland J, Kim R, Wong WC and Weinstein JN: SpliceSeq: A resource for analysis and visualization of RNA-Seq data on alternative splicing and its functional impacts. Bioinformatics 28: 2385-2387, 2012.

27. Lex A, Gehlenborg N, Strobelt H, Vuillemot R and Pfister $H$ : UpSet: Visualization of intersecting sets. IEEE Trans Vis Comput Graph 20: 1983-1992, 2014.

28. Wu G, Feng X and Stein L: A human functional protein interaction network and its application to cancer data analysis. Genome Biol 11: R53, 2010.

29. Liu X, Si W, Liu X, He L, Ren J, Yang Z, Yang J, Li W, Liu S, Pei F, et al: JMJD6 promotes melanoma carcinogenesis through regulation of the alternative splicing of PAK1, a key MAPK signaling component. Mol Cancer 16: 175, 2017.

30. Zhang P, Feng S, Liu G, Wang H, Fu A, Zhu H, Ren Q, Wang B, $\mathrm{Xu}$ X, Bai $\mathrm{H}$ and Dong C: CD82 suppresses CD44 alternative splicing-dependent melanoma metastasis by mediating U2AF2 ubiquitination and degradation. Oncogene 35: 5056-5069, 2016.
31. Furney SJ, Pedersen M, Gentien D, Dumont AG, Rapinat A, Desjardins L, Turajlic S, Piperno-Neumann S, de la Grange P, Roman-Roman S, et al: SF3B1 mutations are associated with alternative splicing in uveal melanoma. Cancer Discov 3: 1122-1129, 2013.

32. Quesada V, Conde L, Villamor N, Ordóñez GR, Jares $\mathrm{P}$, Bassaganyas L, Ramsay $\mathrm{AJ}$, Beà $\mathrm{S}$, Pinyol $\mathrm{M}$, Martínez-Trillos A, et al: Exome sequencing identifies recurrent mutations of the splicing factor SF3B1 gene in chronic lymphocytic leukemia. Nat Genet 44: 47-52, 2011.

33. Biankin AV, Waddell N, Kassahn KS, Gingras MC, Muthuswamy LB, Johns AL, Miller DK, Wilson PJ, Patch AM, $\mathrm{Wu}$ J, et al: Pancreatic cancer genomes reveal aberrations in axon guidance pathway genes. Nature 491: 399-405, 2012.

34. Pan Q, Shai O, Lee LJ, Frey BJ and Blencowe BJ: Deep surveying of alternative splicing complexity in the human transcriptome by high-throughput sequencing. Nat Genet 40: 1413-1415, 2008.

35. Baty F, Klingbiel D, Zappa F and Brutsche M: High-throughput alternative splicing detection using dually constrained correspondence analysis (DCCA). J Biomed Inform 58: 175-185, 2015.

36. Oltean S and Bates DO: Hallmarks of alternative splicing in cancer. Oncogene 33: 5311-5318, 2014.

37. Das $\mathrm{S}$ and Krainer AR: Emerging functions of SRSF1, splic- ing factor and oncoprotein, in RNA metabolism and cancer. Mol Cancer Res 12: 1195-1204, 2014.

38. Díaz R, Peña C, Silva J, Lorenzo Y, García V, García JM, Sánchez A, Espinosa P, Yuste R, Bonilla F and Domínguez G: p73 isoforms affect VEGF, VEGF165b and PEDF expression in human colorectal tumours. VEGF165b downregulation as a marker for poor prognosis. Int J Cancer 123: 1060-1067, 2008.

39. Bates DO, Cui TG, Doughty JM, Winkler M, Sugiono M, Shields JD, Peat D, Gillatt D and Harper SJ: VEGF165b, an inhibitory splice variant of vascular endothelial growth factor, is down-regulated in renal cell carcinoma. Cancer Res 62: 4123-4131, 2002.

This work is licensed under a Creative Commons Attribution-NonCommercial-NoDerivatives 4.0 International (CC BY-NC-ND 4.0) License. 